\title{
Role of Tranexamic Acid in Conservative Treatment of Patients with Epidural Hematoma
}

\author{
Parviz Samadi-Motlagh 1*, Mohammad Shimia ${ }^{1}$, Moslem Shakeri ${ }^{1}$, Azad Mohammadzadeh ${ }^{1}$, Firooz \\ Salehpour ${ }^{1}$, Atta Mahdkhah ${ }^{1}$, Farhad Mirzaiee ${ }^{1}$ \\ ${ }^{1}$ Department of Neurosurgery, Tabriz University of Medical Sciences, Tabriz, East Azerbaijan, Iran \\ * Corresponding Author Address: Department of Neurosurgery, Tabriz University of Medical Sciences, Tabriz, East Azerbaijan, Iran. \\ Tel: +984133340830. E-mail:parviz_sm1550@yahoo.com \\ Article Type: Clinical Trial \\ Received: September 9, 2016, Last Revised: December 11, 2016, Accepted: December 17, 2016, Published: December 30, 2016
}

\section{Abstract}

Introduction: Traumatic brain injury is considered as the major cause of mortality and hospitalization of about ten million people across the globe. Most of these patients are young and active people suffering disabilities. Tranexamic acid therapy is commonly used to reduce blood loss and need for blood transfusions in surgical cases. This study aimed at investigating the effect of tranexamic acid drug in patients with epidural hematoma.

Methods \& Materials/Patients: In a clinical trial study, 100 patients with acute epidural hematoma diagnosed with traumatic brain injury were enrolled. The patients in two groups were matched for age and sex. We treated 50 patients with tranexamic acid drug that were compared with 50 patients in the control group. Hematoma size variations (diameter and volume) between the two groups were studied.

Results: We studied 100 patients with traumatic brain injury with a mean age of $34.2 \pm 7.9$. Of the patients, $69 \%$ were male, and $31 \%$ were female. Hematoma occurrence rate was $33 \%, 39 \%$, and $28 \%$ in parietal, frontal, and temporal region, respectively. Hematoma diameter was $3.71 \pm 1.8 \mathrm{~mm}$ after treatment with tranexamic acid, and $4.03 \pm 1.6 \mathrm{~mm}$ in the control group. There was no statistically significant difference between the two groups. The volume of hematoma was $8.04 \pm 3.2 \mathrm{cc}$ in patients treated with tranexamic acid, and $10.4 \pm 3.7 \mathrm{cc}$ in the control group. There was statistically significant difference between the two groups.

Conclusion: According to our study, tranexamic acid drug can be effective on bleeding control in patients with acute epidural hematoma. The role of these drugs in elective surgeries has been proven. In cases of brain trauma, they can be useful and improve the prognosis of patients.

Keywords: Tranexamic Acid; Acute Epidural Hematoma; Brain Trauma; Cerebral Hemorrhage

Please cite this paper as: Samadi-Motlagh P, Shimia M, Shakeri M, Mohammadzadeh A, Salehpour F, Mahdkhah A, Mirzaiee F. Role of Tranexamic Acid in Conservative Treatment of Patients with Epidural Hematoma. Iran. J. Neurosurg. 2016;2(3):8-10

\section{Introduction}

Traumatic epidural hematoma (EDH) is one of the most common disorder in neurosurgery with high morbidity and mortality [1]. Of all the total brain injuries, 2.7 to $11 \%$ are EDH and large hematomas lead to higher mortality and morbidity, so immediate control is necessary to obstruct such bleedings [2]. Reported mortality rate of EDH is $20 \%$ [3]. Most patients are men under 45 years old whose injuries cost much to patient and society $[3,4]$. Laceration of middle meningeal artery is the most common cause of EDH. If the bleeding is not controlled, the result will be increased brain pressure, herniation and death [5]. The main treatment of EDH is surgery, but in patients with no such a need, some conservative methods are done, like some drugs. Antifibrinolytic drugs such as tranexamic acid (TXA) have essential role in reducing hematoma volume and mortality in major surgeries and traumatic patients without any important complication [2,3]. Most studies have confirmed that TXA is a safe and effective drug in acute bleeding events to control volume and size of hematoma [5-7]. The goal of this study was to evaluate the role of TXA in traumatic EDH patients admitted to Imam Reza Hospital of Tabriz University of Medical Sciences (North West of Iran). This study has been registered as a clinical trial in www.irct.ir (IRCT201011202854N6) and has been registered and confirmed by ethical committee of the university (No: 5.4. 5898).

\section{Methods and Materials/Patients}

In this study, 100 patients with head trauma, EDH with maximum diameter lesser than $10 \mathrm{~mm}$, aged between 18 to 75 years old, GCS $=15$, written participation consent form were randomly selected. All patients lacking the upper criteria; GCS $<13$, requiring surgery, having $\mathrm{EDH}$ in occipital region, positive history of major disorder, positive history of antifibrinolytic consumption were excluded. All 100 patients admitted to Imam Reza Hospital from April 2014 to March 2016 underwent the intervention. Before intervention, all of these criteria were checked for all patients, and they were randomly divided into two groups; i.e. group A (cases) and 
group B (control). In this study, the effect of TXA on conservative treatment of EDH in traumatic patients are discussed. This study was randomized-single blind with use of placebo. In two parallel groups of 50 study cases and controls, men and women aged 18 to 75 years old with diagnosis of EDH (with no associated severe traumatic injuries) in Tabriz Imam Reza hospital, not requiring surgical intervention, were selected. The intervention group received one gr ampule TXA as loading dose and then one gr every eight hours for two days, but control group received placebo. After two days, spiral brain CT scan was performed for all patients, and the two groups for some parameters such as the rate of change in EDH volume and diameter, level of consciousness and the rate of need for surgery before and after intervention were compared.

\section{Results}

We studied 100 patients with traumatic brain injury with a mean age of $34.2 \pm 7.9$ years old. Of the patients, $69 \%$ were male, and $31 \%$ were female. Hematoma occurrence rate was $33 \%, 39 \%$, and $28 \%$ in parietal, frontal, and temporal region, respectively. Hematoma diameter was $3.7 \pm 1.8 \mathrm{~mm}$ after treatment with TXA, and $4.03 \pm 1.6 \mathrm{~mm}$ in the control group. There was no statistically significant difference between the two groups (Table 1). The volume of hematoma was $8.04 \pm 3.2 \mathrm{cc}$ and $10.4 \pm 3.7 \mathrm{cc}$ in TXA-treated patients and control group, respectively. A statistically significant difference existed between the two groups (Figures 1-3).

Table 1. Comparison of Volume and Diameter of EDH in Brain C.T Scan in Two Groups

\begin{tabular}{|c|c|c|c|c|c|}
\hline \multirow{2}{*}{} & \multicolumn{2}{|c|}{ Control } & \multicolumn{2}{c|}{ Intervention } & $\begin{array}{c}\text { P } \\
\text { Intergroup }\end{array}$ \\
\cline { 2 - 6 } & After & Before & After & Before & \\
\hline Diameter & $4.3 \pm 1.6$ & $3.7 \pm 1.8$ & $4.4 \pm 1.6$ & $3.9 \pm 2$ & 0.85 \\
\hline P & \multicolumn{2}{|c|}{0.22} & \multicolumn{2}{c|}{0.15} & \\
\hline Volume & $10.4 \pm 3.7$ & $8 \pm 3.4$ & $10.7 \pm 3.9$ & $8.1 \pm 3.7$ & 0.001 \\
\hline P & \multicolumn{2}{|c|}{0.007} & \multicolumn{2}{c|}{0.21} & \\
\hline
\end{tabular}

\section{Discussion}

Traumatic brain injury is the most common cause of mortality and morbidity in young patients, as observed in our study in patients with mean age of 33 years old. Vehicle accident has been reported as the most common cause of trauma. Most of these victims are under 40 years old, leading to remarkable costs for patients and society [8]. Some patients need surgery intervention; however, many do not require surgery. These patients are treated with conservative proceedings such as antifibrinolytic agents. TXA, an antifibrinolytic drug, reported to be safe in most prior studies, had no serious side effects in our study $[9,10]$. The highest effectiveness of TXA is at first four hours after trauma and injection, so in our study, we started TXA administration immediately after diagnosis of EDH in patients [8]. In a study by Johansson et al. (CRASH 2), TXA was useful in reducing bleeding volume and mortality [11]. In the control group of our study, hematoma volume had a significant increase, whereas hematoma volume did not increase significantly in the intervention group. In both groups, hematoma diameter had no significant change. In a study by Morrison et al. in USA army, with using TXA in traumatic patients, mortality was reduced, and TXA was effective in EDH and reduced mortality and morbidity in our study. In another study by Perel et al., bleeding volume reduced from $5.9 \mathrm{~mm}$ to $4.8 \mathrm{~mm}$ by administration of TXA in intervention group, and new bleeding site appeared in 11\% of patients in intervention group, and $16 \%$ in control group [12]. In control group of the this study, hematoma volume increased from $8 \pm 3.4 \mathrm{cc}$ to $10.4 \pm 3.7 \mathrm{cc}$, and P-value was 0.007 that was statistically significant, while in the intervention group, P-value was 0.21 which was not statistically significant. The difference between hematoma diameters was not significant in the two groups; P-value was 0.22 and 0.15 in the control group and intervention group, respectively. It means that TXA was effective in maintaining and reducing hematoma volume, but had no significant effect on hematoma diameter.

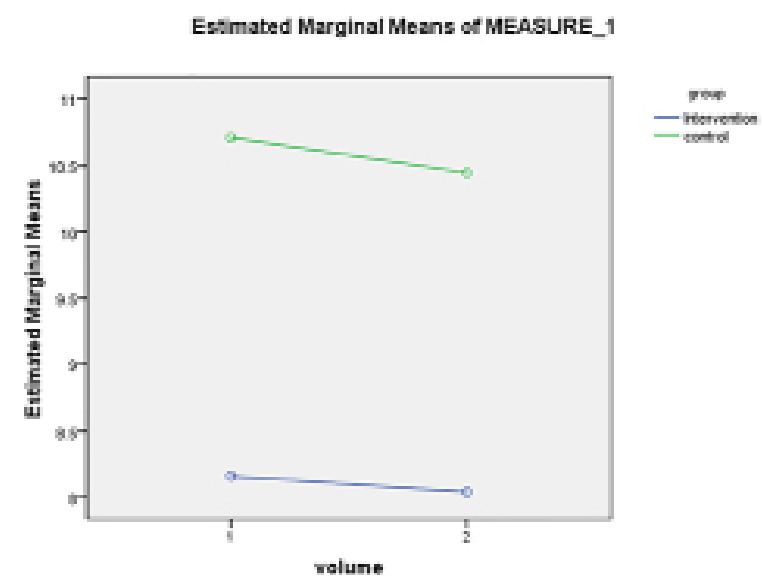

Figure 1. Changes in Volume of Hematoma between Two Groups Estimated Marginal Means of MEASURE_1

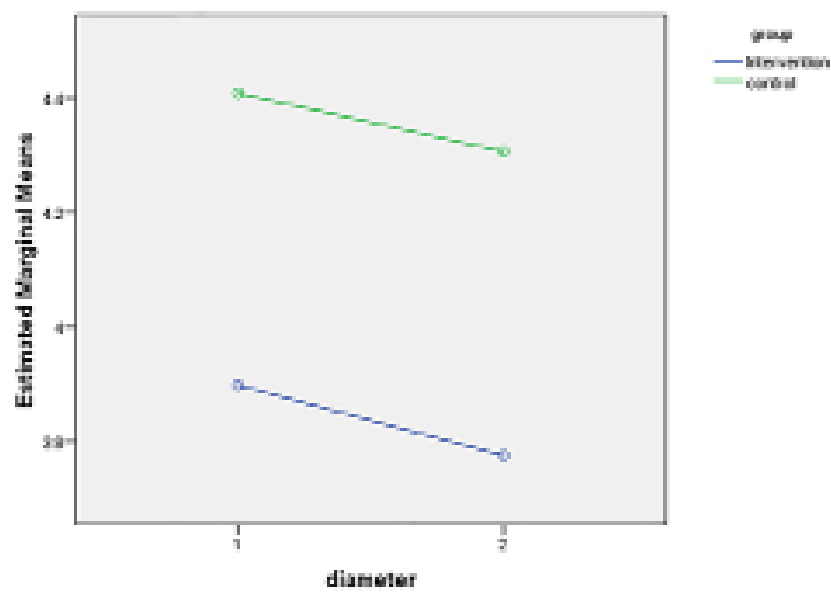

Figure 2. Changes in Diameter of Hematoma between Two Groups

\section{Conclusion}

According to our study, TXA drug can have a critical role in controlling bleeding in EDH patients. The role of this drug in elective surgeries has been proven, and in cases of brain injury could be useful and improve the prognosis of patients.

\section{Funding}

None. 


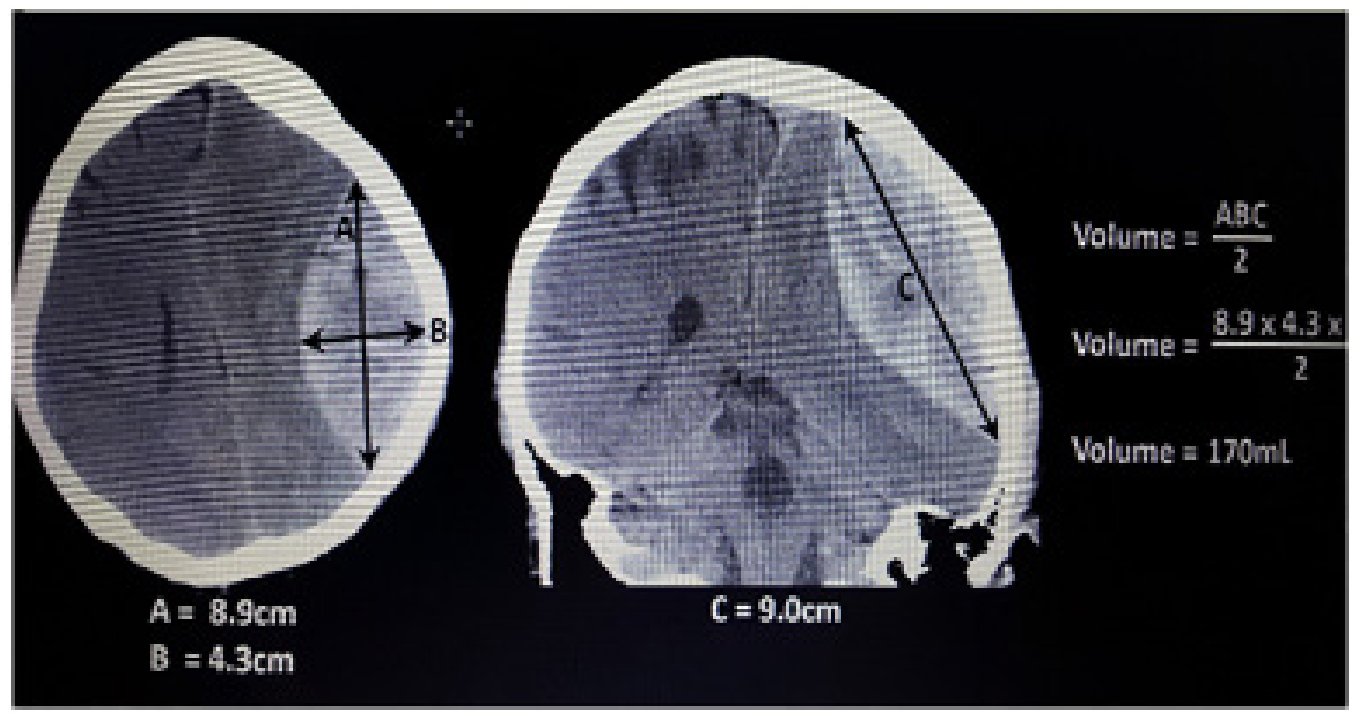

Figure 3. Calculating the Volume and Diameter of Epidural Hematoma in Patients

\section{Conflicts of Interest}

The authors declare no conflict of interest.

\section{Authors' Contribution}

Conception and Design: Parviz Samadi-Motlagh, Mohammad Shimia, Moslem Shakeri. Data Collection: Azad Mohammadzadeh, Atta Mahdkhah, Farhad Mirzaiee. Drafting the Article: Firooz Salehpour, Azad Mohammadzadeh. Critical Revising the Article: Atta Mahdkhah. Reviewed Submitted Version of Manuscript: all authors. Approved the Final Version of the Manuscript: all authors.

\section{References}

1. Cheung PS, Lam JM, Yeung JH, Graham CA, Rainer TH. Outcome of traumatic extradural haematoma in Hong Kong. Injury. 2007;38(1):76-80.

2. Becker DP, Miller JD, Ward JD, Greenberg RP, Young HF, Sakalas R. The outcome from severe head injury with early diagnosis and intensive management. Journal of neurosurgery. 1977;47(4):491-502.

3. Thurman D, Guerrero J. Trends in hospitalization associated with traumatic brain injury. Jama. 1999;282(10):954-7.

4. Etemadrezaie H, Zabihian S, Baharvahdat H, Ganjeifar B. Acute Epidural Hematoma after Ventriculoperitoneal Shunt Insertion: a Case Report. Iranian Journal of Neurosurgery. 2015;1(3).

5.Bricolo AP, Pasut LM. Extradural Hematoma: Toward Zero Mortality: A Prospective Study. Neurosurgery. 1984;14(1):8-12.

6. Rodgers A, MacMahon S. Systematic underestimation of treatment effects as a result of diagnostic test inaccuracy: implications for the interpretation and design of thromboprophylaxis trials. Thrombosis and haemostasis. 1995;73(2):167-71.

7. Horrow JC, Van Riper DF, Strong MD, Grunewald KE, Parmet JL. The doseresponse relationship of tranexamic acid. The Journal of the American Society of Anesthesiologists. 1995;82(2):383-92.

8. Ker K, Edwards P, Perel P, Shakur H, Roberts I. Effect of tranexamic acid on surgical bleeding: systematic review and cumulative meta-analysis. Bmj. 2012;344:e3054

9. Crescenti A, Borghi G, Bignami E, Bertarelli G, Landoni G, Casiraghi GM, et al. Intraoperative use of tranexamic acid to reduce transfusion rate in patients undergoing radical retropubic prostatectomy: double blind, randomised, placebo controlled trial. BMJ. 2011;343:d5701.

10. Alvarez JC, Santiveri FX, Ramos I, Vela E, Puig L, Escolano F. Tranexamic acid reduces blood transfusion in total knee arthroplasty even when a blood conservation program is applied. Transfusion. 2008;48(3):519-25.

11. Williams-Johnson J, McDonald A, Strachan GG, Williams E. Effects of tranexamic acid on death, vascular occlusive events, and blood transfusion in trauma patients with significant haemorrhage (CRASH-2): a randomised, placebo-controlled trial. West Indian Medical Journal. 2010;59(6):612-24.

12. Collaborators C-. Effect of tranexamic acid in traumatic brain injury: a nested randomised, placebo controlled trial (CRASH-2 Intracranial Bleeding Study). BMJ. 2011;343:d3795.

\section{Comments}

There is growing body of evidence on the role of tranexamic acid (TXA) in conservative treatment of traumatic or non-traumatic intracranial hematomas. In this study, the authors attempted to provide new evidence for its role in treatment of epidural hematoma (EDH). For this reason, a group of patients selected for conservative treatment of EDH were given TA and showed less hematoma volume expansion compared with the control group over time.

Although, the study is a prospective, randomized, and controlled trial, it harbours major drawbacks which preclude it from being classified as the highest level of evidence. Many details of the methodology are missed including but not limited to age distribution, coagulation profile, randomization method, and biostatistical tests. Patient outcome at discharge or their follow-up status is not reported as well. Regarding the results, if data are presented as mean \pm confidence interval as is usual, there are no statistically significant differences between the groups. Yet, even if we accept a difference, it is only statistically as the difference in expansion between the two groups was less than 0.5 cc $(10.4 \pm 3.7$ vs. $10.7 \pm 3.9)$ which is clinically negligible. Also, we should notice that the authors have used ABC method for "estimating" the hematoma volume which is not accurate for research purposes and preferably, a scientific software (e.g. Image J) should have been used to "calculate" the volume.

Another important point is that we have evidence showing the importance of timing between the accident and the first brain CT scan indicating that the shorter this time, the higher the chance of developing new hematoma or experiencing volume expansion in an existing one. This was again neglected by the study as there is no information on the average time between the happening of trauma and the first CT scan. Finally, we should bear in mind that the goal of such treatment is to control hematoma expansion to prevent further surgeries/interventions in conservatively treated patients. However, the number of patients deteriorated clinically (e.g. developing intracranial hypertension symptoms such as nausea, vomiting, drowsiness, headache, etc), required surgery, or even died in each group is not reported. Taking all mentioned above into consideration, it is impossible to draw conclusion whether or not this intervention may affect our decisions or patient outcome according to the current study.

Mohsen Nouri, Board of Directors, Asian Congress of Neurological Surgeons (ACNS), Gundishapour Academy of Neuroscience, Ahvaz, Iran 